\title{
Monomolecular Layers of Pyrene as a Sensor to Dicarboxylic Acids
}

Lining Gao, Yu Fang, ${ }^{*}$ Xiangpeng Wen, Yuangang Li, and Daodao Hu

School of Chemistry and Materials Science, Shaanxi Normal University, Xi' an 710062, China

E-mail: yfang@ @nnu.edu.cn

${ }^{1} \mathrm{H}$ NMR spectra of PSC in $\mathrm{CHCl}_{3}-d$ and PSEDA in DMSO- $d$ recorded on a Bruker AM400 NMR spectrometer
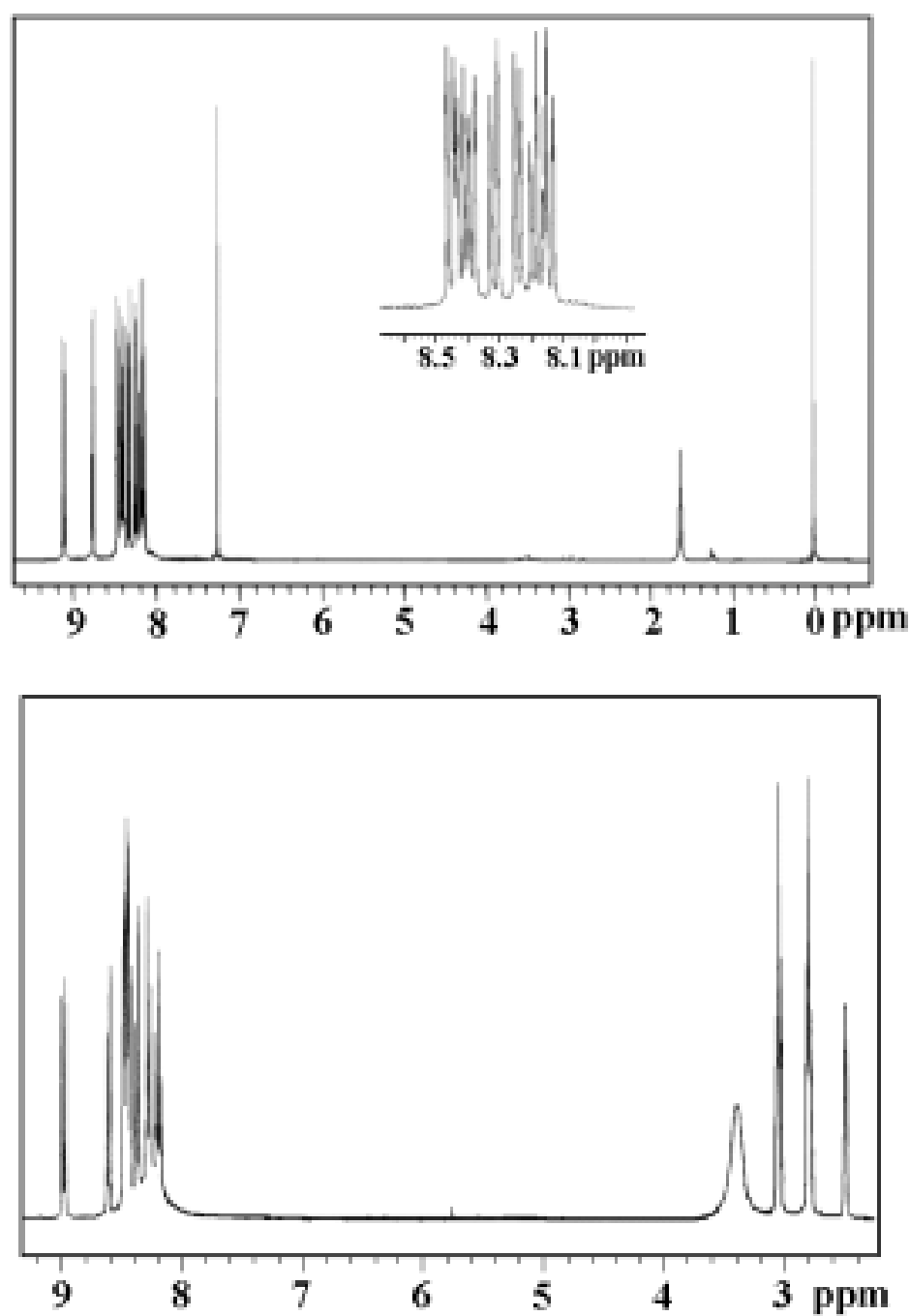

${ }^{1} \mathrm{H}$ NMR spectra of PSC $\left(\mathrm{CHCl}_{3}-d\right)$ (top) and PSEDA (DMSO- $d$ ) (bottom) 